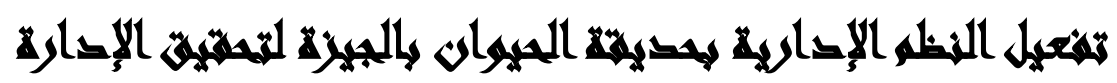

\section{المئئية}

[rq]

خالد حسين أحمد(')- محمد سالم محمود سالم(')- ناهد إبراهيم محمد متولي

( ) كلية التجارة، جامعة عين شمس احمد (1)

\section{المستخلص}

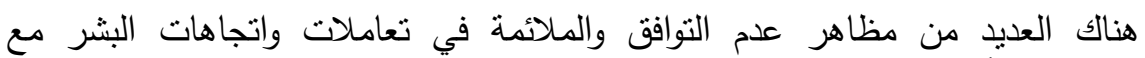

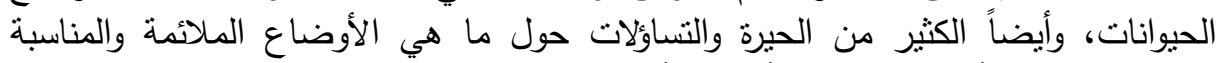

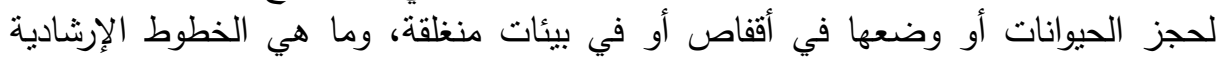

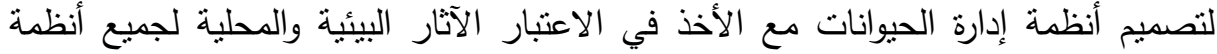

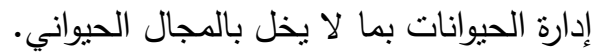
هدفت الدراسة تفعيل نظام الإدارة البيئية في حديقة الحئية الحيوان بالجيزة وتحديد أوجه القصور

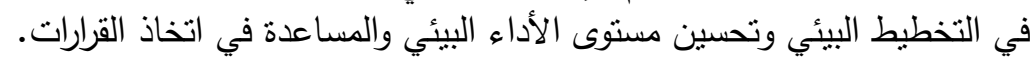

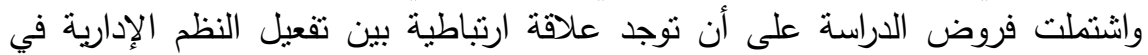

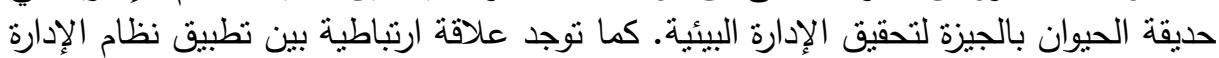

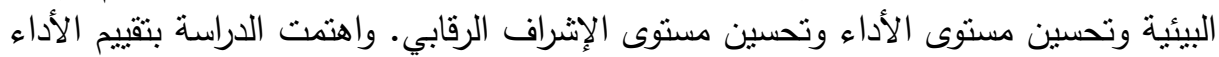
البيئي الحالي لنظام الإدارة البيئية وتحديد أوجه القصور في تطبيق نظام الإدارة البيئية.

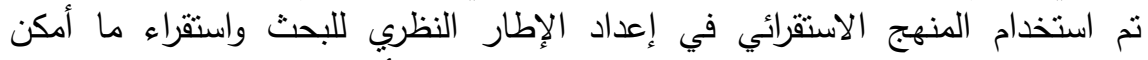

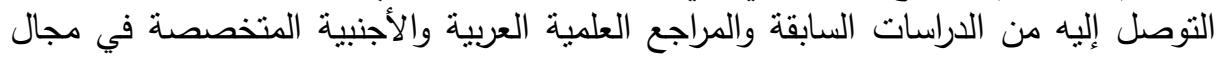

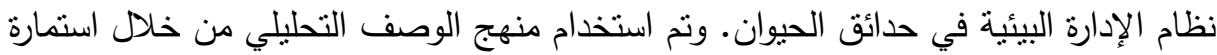

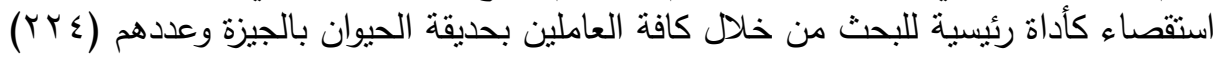
فرد. تقتصر حدود الدراسة على الحدود المكانية وهي حديقة الحيوان بالجيزة والأفراد (كافة (كافية العاملين بالحديقة - إداريين - بيطريين).

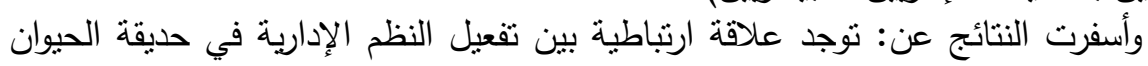

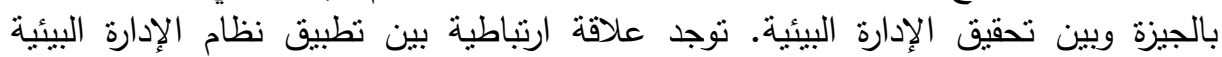

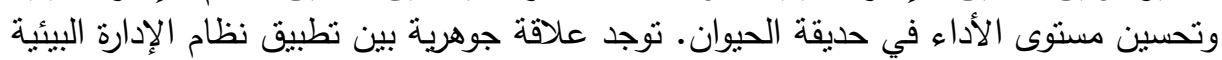
وتحسين مستوى الإثراف الرقابي. 


\section{ranadl}

هناك العديد من مظاهر عدم التوافق والملائمة في تعاملات واتجاهات البشر مع

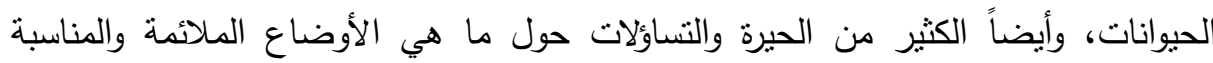

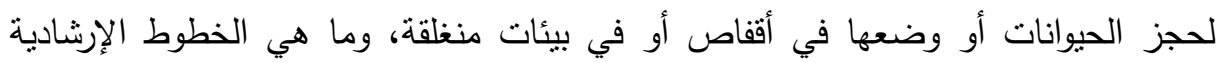
لتصميم أنظمة إدارة الحيوانات مع الأخذ في الاعنبار الآثار البيئية والمحلية لجميع أنظمة فئية

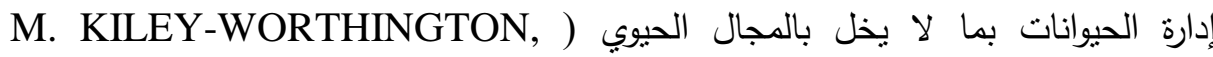
2012). بما أن الإنسان عرف الإدارة منذ وجدت المجتمعات الإنسانية على هذه البسيطة،

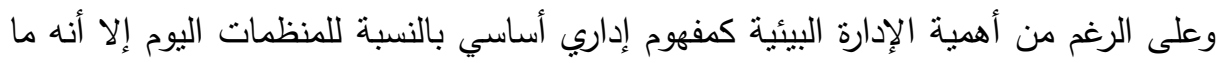

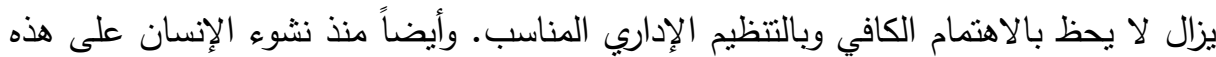

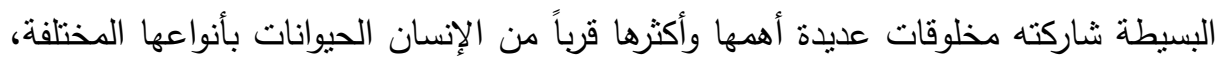

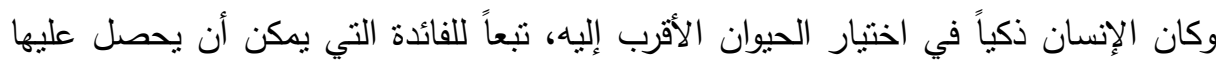

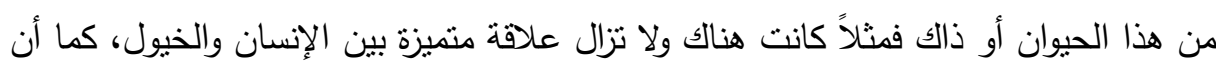
الفيل له حضوره في حياة الإنسان. في المقابل هناك أضرار قد تلحقها الحيوانات بالإنسان، لاسيما المفترسة منها، لهذا وضع الإنسان قيوداً على حرية وحركة هذه الحيوانات. لذا يجب عند دراسة تقييم احتياجات الحيوان السلوكية مراعاة الأهمية البيئية واحتباجات لأجناس على اختلافها وأيضاً الاحتياجات الفردية لكل نوع، وسيساعد في ذلك أن أن الإدارة (العامة، البيئية) اليوم أصبحت علم منطور، أنى بمبتكرات كثيرة لتسجيل التحركات الداخلية ضمن المؤسسة أو المنظمة (سواء كانت حكومية أو أهلية) والوصول إلى أقصى استخلاتلال

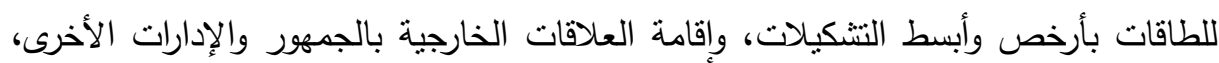
وبالإمكان الاستفادة بتوسع أساليب الإدارة الحديثة وعلى الأخص أساليب إدارة البيئة (قلنش

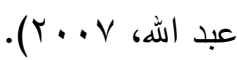




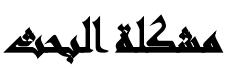

تواجه المؤسسات الحكومية في جمهورية مصر العربية وحديقة الحيوان بصفة خاصة مجموعة من أوجه القصور تحد من كفاءة وفاعلية الأداء في تلك المؤسسات الهامة مما يجعل وله عملية تقييم الأداء بها يتسم أيضاً بعدم الموضوعية، ويحاول الباحثون من خلال ذلك البحث تتاول بعض أوجه القصور في حديقة الحيوان بالجيزة لكي يمكن اتخاذ ما يلزم من إجراءات

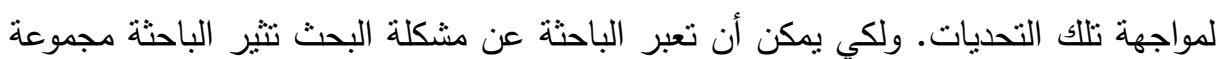
من التساؤلات لعلها تضع النقط على الحروف فيما يتعلق بمشكلة البحث (عطية جا...r،

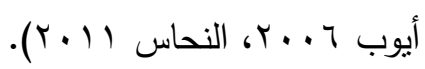

\section{أسريلا المهمش}

- هل مستوى الأداء البيئي في حديقة الحيوان بوضعه الراهن يساعد ما الوضع الحالي لمستوى الأداء البيئي وعلاقته بتحقيق نظام الإدارة البيئية ؟ - ما الوضع الحالي للإثراف الرقابي البيئي بوضعه وعلاقته بتحقيق أهداف نظام الإدارة البيئية:

- ما مدى تفعيل النظم الإدارية وعلاقته بتحقيق الإدارة البيئية.

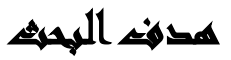

يحاول الباحثون من تلأك الدراسة تحقيق الأهداف الآتية: الههف الرئيسي: تفعيل نظام الإدارية البيئية في حديقة الحيوان بالجيزة.

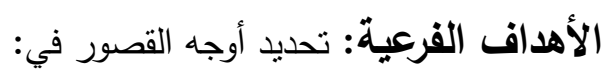
- تحسين مستوى الأداء البيئي.

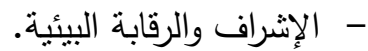


مجلة العلوم البيئية

معهد الدراسات والبحوث البيئية - جامعة عين شمس لئس

\section{هروغر اللهحثي}

الفرض الرئيسي: توجد علاقة ارتباطية ذات دلالة بين تفعيل النظم الإدارية في حديقة الحيوان بالجيزة لتحقيق الإدارة البيئية. وينبثق منه الفروض الفرعية الآتية: - توجد علاقة ارتباطية ذات دلالة بين تطبيق نظام الإدارة البيئية وتحسين مستوى الأداء في

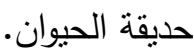
- توجد علاقة ارتباطية ذات دلالة بين تطبيق نظام الإدارة البيئية وتحسين مستوى الإثراف

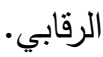

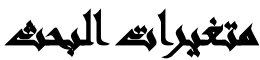

$$
\begin{aligned}
& \text { المتغير المستقل: } \\
& \text { - تحسين مستوى الأداء. } \\
& \text { - تحسين مسنوى الإثراف الرقابي. } \\
& \text { - النظم الإدارية. } \\
& \text { المتغير التابع: } \\
& \text { - الإدارة البيئية. }
\end{aligned}
$$

\section{Aatl}

الحدود المكانية: داخل حديقة الحيوان وإظهار أهمية تفعيل نظام الإدارية البيئية. الأفراد: مدير الإدارة المركزية لحدائق الحيوان والحياة البرية - المديرين والمشرفين - كافة العاملين بالحديقة (العاملين الإداريين - الأطباء البيطريين). 


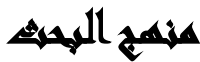

القسم الأول: المنهج الاستقرائي: حيث اعتمد البحث على الدنهج الاستقرائي في إعداد

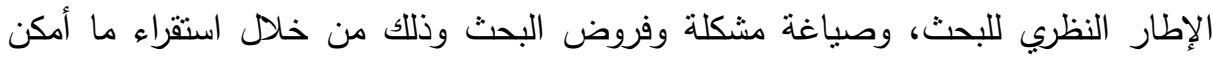
التوصل إليه من المراجع العلية العربية والأجنبية المتخصصة في مجال نظام الإدارة الييئية في حدائق الحيوان. القسم الثاني: المنهج التحليلي: اعتدت الدراسة على الأسلوب الوصفي التحليلي

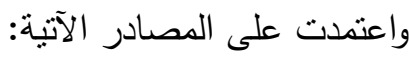
مصادر ثانوية: استخدام المراجع والمصادر المتوفرة في الدكتبات وذلك لبناء الإطار النظري لهذه الدراسة ومنها الكتب والدراسات السابقة والمقالات. مصادر أولية: دراسة تطبيقية على حديقة الحيوان.

\section{أهمية الهمهي}

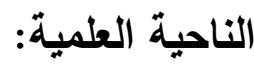
- تقييم الأداء البيئي الحالي لنظام الإدارة البيئية في حديقة الحيوان. - تحديد أوجه القصور في تطبيق نظام الإدارة البيئية.

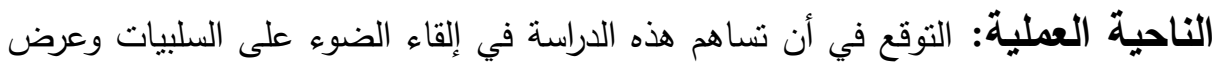
بعض التوصيات للحد من هذه السلبيات.

\section{مسطلحاهي المهنه}

النظم الإدارية: يقصد بالنظام الإداري ذلك الكيان المتكامل الذي يمثل الإطار العام للعمل الإداري في مجتمع ما. والنظام الإداري Administrative System يمثل تركيباً معقداً ينكون من أجزاء متعاونة ومتفاعلة بحيث تتحدد الكفاءة الكلية للنظام في ضوء الإداءئ التفاعلات بين أجزاءه وتختلف باختلاف مستويات الكفاءة لكل منها. إن هذا التصور للنظام الإداري بعتبر أن

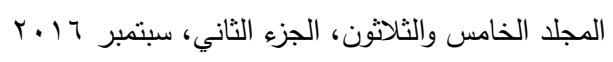


العمل الإداري في مجتمع ما (أو في أي جزء من أجزاء المجتمع) هو محصلة للتفاعل بين

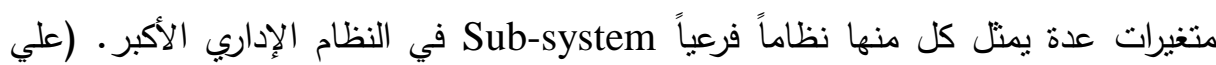

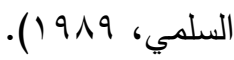
الإدارة البيئية: هو نظام يمكن المنظمة من التحكم في تأثيرات أنشطتها ومنتجاتها وخدماتها على البيئة الطبيعية، وذلك بالالتزام بمعايير فنية وصناعية وتجارية وتحصل المنشأة المطبقة لنظام الإدارة البيئية على شهادة الأيزو الدولية (السيد عيد نايل، V . . ب).

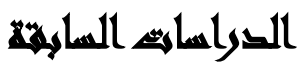

دراسة البناني (1 ( + ب): من أهم أهداف هذه الدراسة تحديد معوقات استخدام النظم

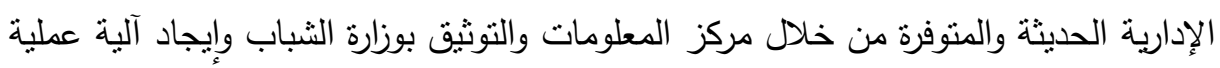

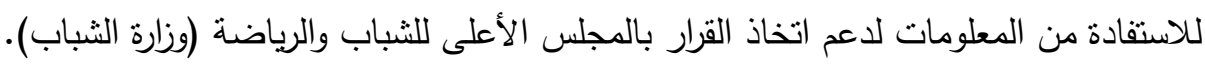

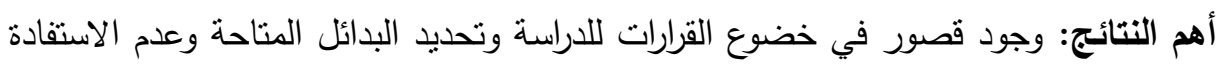
من المعلومات المتوفرة بمركز المعلومات والتوثيق ودعم اتخاذ القرار .

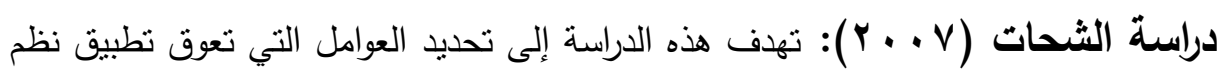

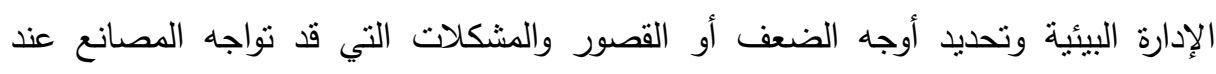
تطبيق نظم الإدارة البيئية ومقارنة بين المناطق الصناعية الجديدة والقديمة لتحديد أوجه الاختلاف بين المنطقتين فيما يتعلق بتطبيق نظم الإدارة البيئية.

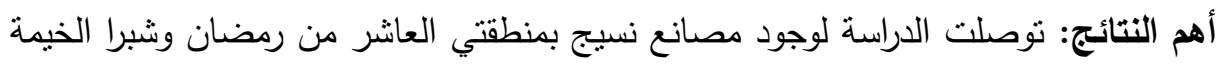

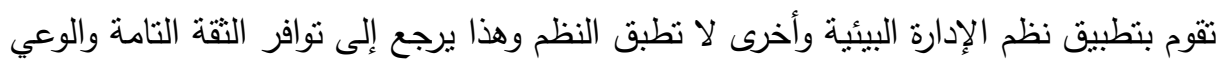

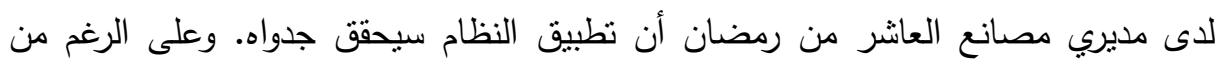

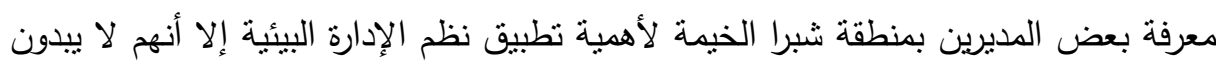

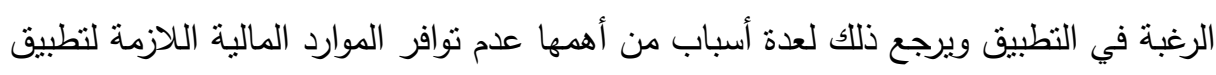




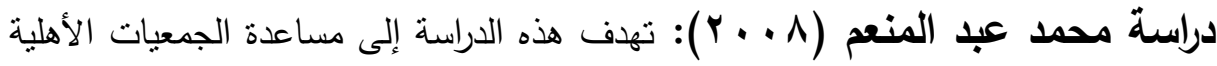

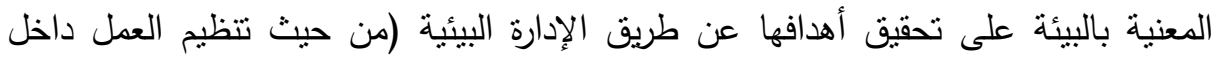

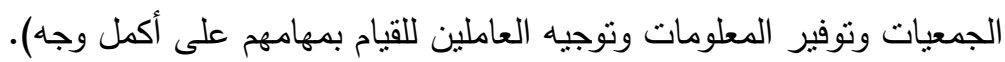

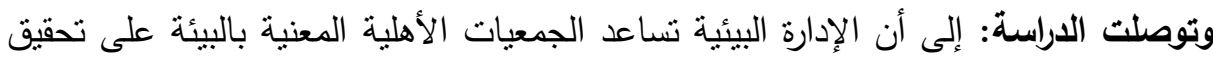

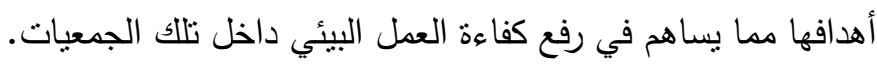

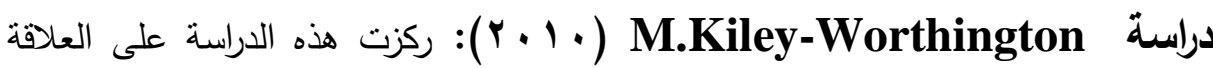
الارتباطية بين الحيوانات بعضها البعض وبين البيئة وأهمية التتوع في استقرار وصيانة الأنظمة البيئية ووضعها في الاعتبار عند تقييم إدارة الحيوان.

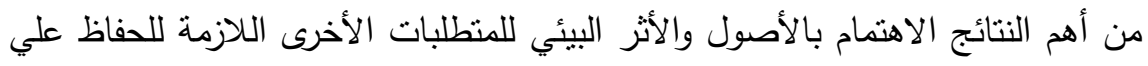

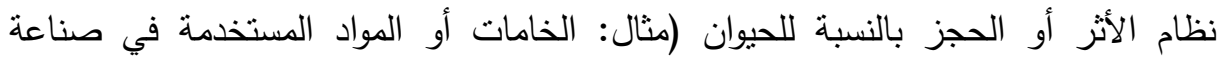
المأوى، الأسوار ، التذفئة .. إلخ). دراسة (Y) Viaro, O; Roberti, F. V. and Bressa, P.M. (11) هدفت هذه الدراسة إلى عمل مشروع يهدف الي توسيع المناقثة حول القضايا البيئية لنقديم

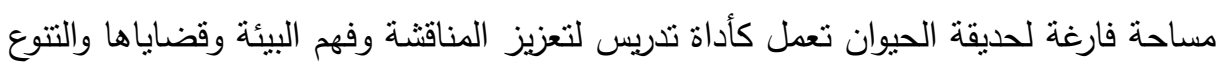

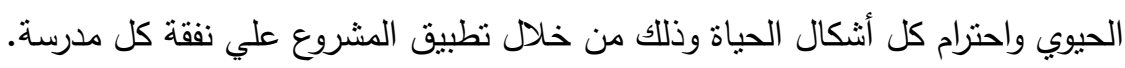

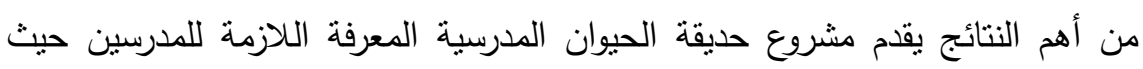
يمكهم بعد ذلك تطبيق المشروع داخل مدارسهم.

\section{اجزاعايت البهمش}

أداة البحث: إعداد استمارتي استبيان على مجموعتين:

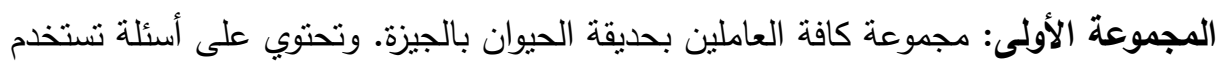

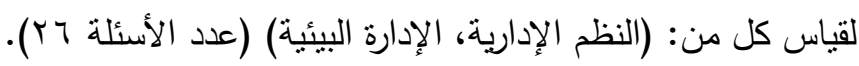


المجموعة الثانية: مجموعة المديرين والمشرفين (إداريين - بيطريين) بحديقة الحيوان بالجيزة.

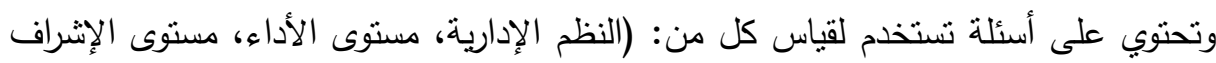
الرقابي) (عدد الأسئلة 0 (1)).

\section{قياس الاعتمادية (ثبات وصدق استمارة الاستبيان):}

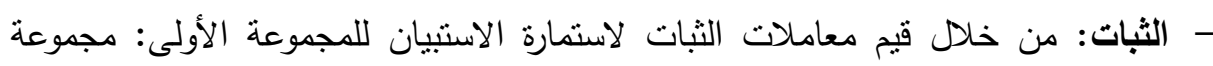

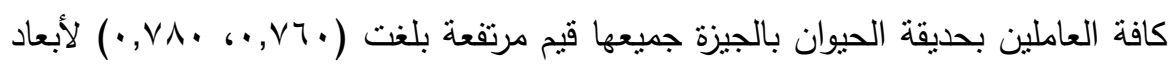

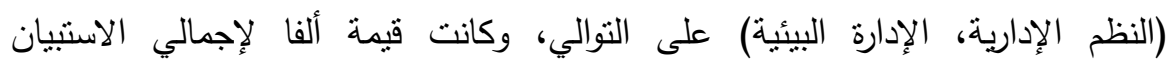

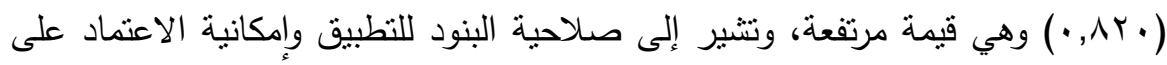
نتائجها والوثوق بها، وكذلك قيم معاملات الثبات لاستمارة الاسنبيان المجموعة الثانية:

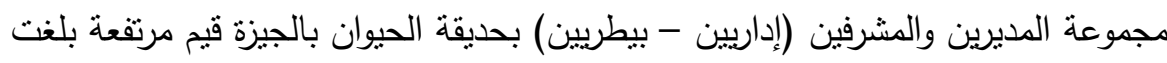

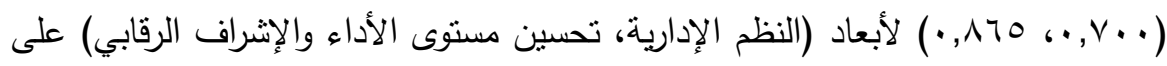

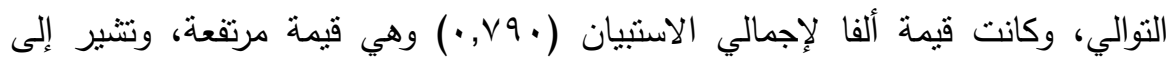
صلاحية البنود للتطبيق وإمكانية الاعتماد على نتائجها والوثنوق بها.

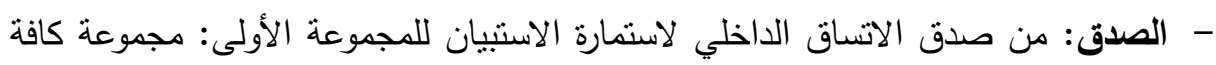

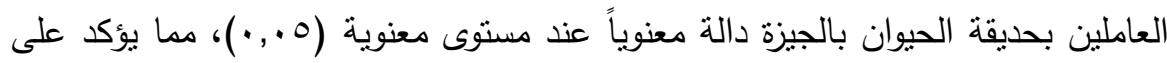

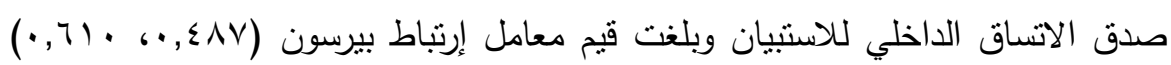
وهي قيم تؤكد على صدق الاستبيان. أما صدق الاتساق الداخلي لأبعاد استمارة الاستبيان للمجموعة الثانية: مجموعة المديرين

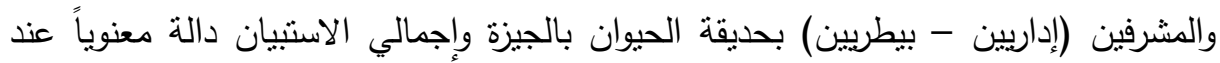

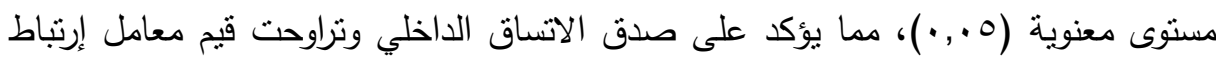

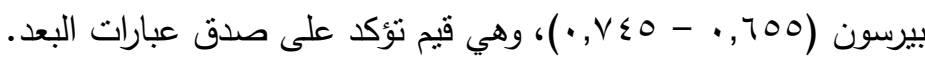




\section{لمنائي الترواسلة}

الفرض الفرعي الأول: توجد علاقة إرتباطية ذات دلالة معنوية بين تطبيق نظام الإدارة البيئية وتحسين مستوى الأداء في حليقة الحيوان بالجيزة. جدول(1): اختبار العلاقة الإرتباطية بين تطبيق نظام الإدارة البيئية وتحسين مستوى الأداء في حديقة الحيوان بالجيزة

\begin{tabular}{|c|c|c|}
\hline مستوى المعنوية & معامل الإرتباط (R) & \\
\hline$\cdot, \ldots 1$ & $\cdot, 077$ & تطبيق نظام الإدارة البيئية وتحسين مستوى الأداء \\
\hline
\end{tabular}

يتضـح من نتائج تحليل الانحدار الخطي البسيط في الجدول السـابق أن قيمـة معامل

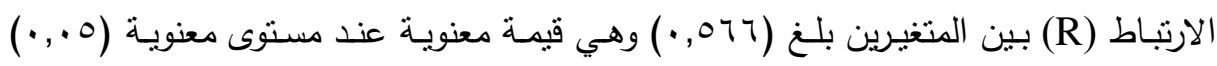
وكانت مستوى المعنويـة للعلاقة (1 +., •) لذا توجد علاقة إرتباطية بين تطبيق نظام الإدارة البيئية وتحسين مستوى الأداء في حديقة الحيوان بالجيزة.

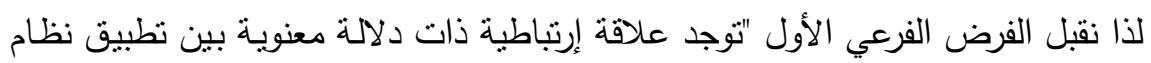
الإدارة البيئية وتحسين مستوى الأداء في حديقة الحيوان بالجيزة ".

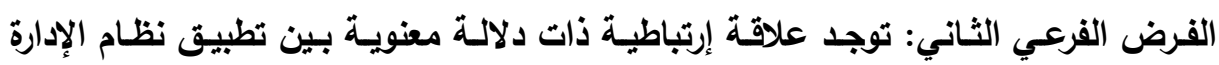
البيئية وتحسين مستوى الإثراف الرقابي. جدول(r): اختبار العلاقة الإرنباطية بين تطبيق نظام الإدارة البيئية وتحسين مستوى الإثراف

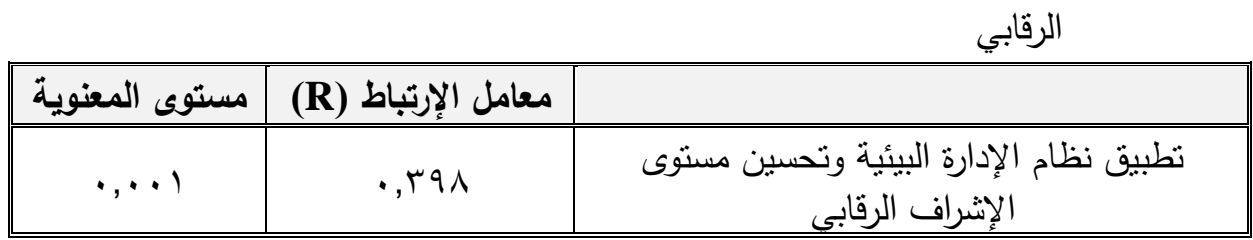

يتضح من نتائج تحليل الانحدار الخطي البسيط في الجدول السابق أن قيمة معامل

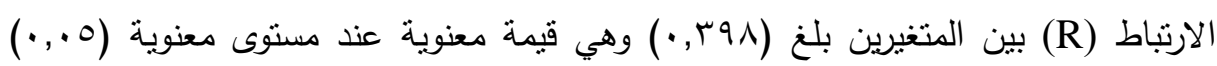
وكانت مستوى المعنوية للعلاقة (1) (.,..) لذا توجد علاقة إرتباطية بين تطبيق نظام الإدارة

$$
\text { البيئية وتحسين مستوى الإشراف الرقابي. }
$$

$$
\text { المجلد الخامس والثلاثون، الجزء الثاني، سبتمبر r } 17
$$


مجلة العلوم البيئية

معهد الدراسات والبحوث البيئية - جامعة عين شمس لهن

لذا نقبل الفرض الفرعي الثاني: "توجد علاقة إرتباطية ذات دلالة معنوية بين تطبيق نظام الإدارة البيئية وتحسين مستوى الإشراف الرقابي".

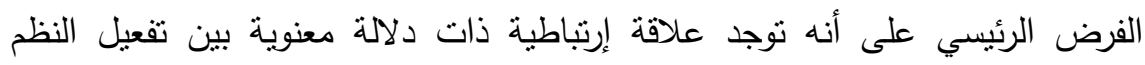
الإدارية في حديقة الحيوان بالجيزة وتحقيق الإدارة البيئية. جدول(ץ): اختبار العلاقة الإرتباطية بين تفعيل النظم الإدارية في حديقة الحيوان بالجيزة وتحقيق الإدارة البيئية

\begin{tabular}{|c|c|c|}
\hline مستوى المعنوية & معامل الإرتباط (R) & \\
\hline$\cdot, \ldots)$ & $\cdot, \varepsilon, 1$ & تفعيل النظم الإدارية في حديقة الحيوان بالجيزة \\
\hline
\end{tabular}

يتضح من نتائج تحليل الانحدار الخطي البسيط في الجدول السابق أن قيمة معامل الارتباط (R) بين المتغيرين بلغ (1 (ـ), •) وهي قيمة معنوية عند مستوى معنوية (0., •)

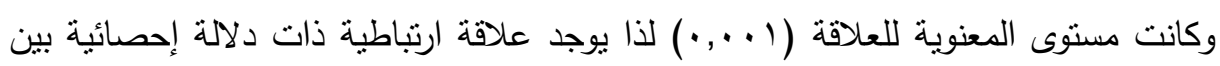
تفعيل النظم الإدارية في حديقة الحيوان بالجيزة وتحقيق الإدارة البيئية. لذا نقبل الفرض الرئيسي: "توجد علاقة إرتباطية ذات دلالة معنوية بين تفعيل النظم الإدارية في حديقة الحيوان بالجيزة وتحقيق الإدارة البيئية".

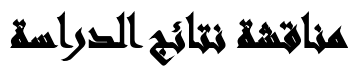

يتضح من نتائج تحليل الانحدار الخطي البسيط أن قيمة معامل الارتباط (R) بين

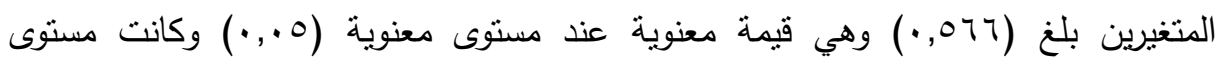
المعنوية للعلاقة (1 (.,.•) لذا توجد علاقة إرتباطية بين نطبيق نظام الإدارة البيئية وتحسين مستوى الأداء في حديقة الحيوان بالجيزة. لذا نقبل الفرض الفرعي الأول: "توجد علاقة إرتباطية ذات دلالة معنوية بين تطبيق نظام الإدارة البيئية وتحسين مستوى الأداء في حديقة الحيوان بالجيزة ". وسوف يؤدي أيضاً تحسين مستوى الأداء روجع حديقة الحيوان منظمة حدائق الحيوان

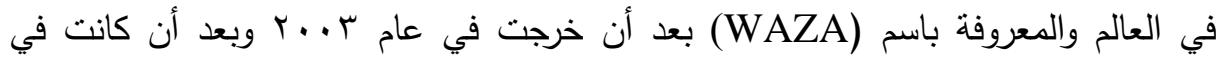
660

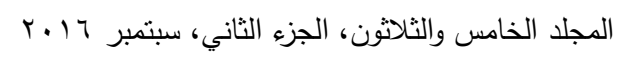


الترنيب الثالث على العالم (elbadil , 2015) ونتيجة تحسين مستوى الأداء في حديقة حيوان

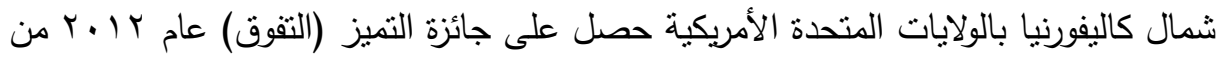
رابطة حدائق الحيوان (Environmental Management System, 2014). يتضح من نتائج تحليل الانحدار الخطي البسيط أن قيمة معامل الارتباط (R) بين

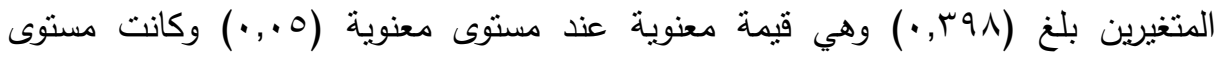
المعنوية للعلاقة (1 .,., لذا توجد علاقة إرتباطية بين نطبيق نظام الإدارة البيئية وتحسين

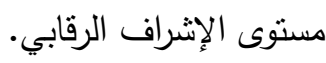
لذا نقبل الفرض الفرعي الثاني: "توجد علاقة إرتباطية ذات دلالة معنوية بين تطبيق نظام الإدارة البيئية وتحسين مستوى الإثراف الرقابي". إن تحسين مستوى الإثراف الرقابي يؤدي إلى مواجهة المشاكل الطارئة مثل الأمراض المعدية حيوانية المصدر (أنفلونزا الطيور) وقد قامت حديقة حيوان بازل بسويسرا بعمل دراسة بلنة بأن الهدف الرئيسي منمثلاً في الحصول على نتائج باثولوجية (أسباب الأمراض)، التاريخ التغذوي ومعلومات عن شجرة العائلة للحيوان (H. Clauss, M. , 2008).

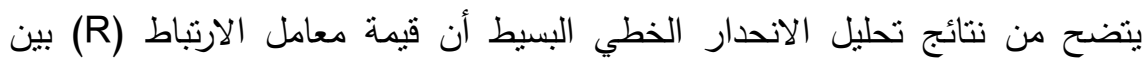

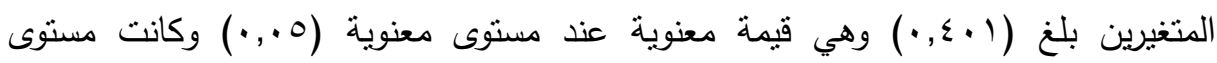
المعنوية للعلاقة (1 ..,·) لذا يوجد علاقة ارتباطية ذات دلالة إحصائية بين تفعيل النظم الإدارية في حديقة الحيوان بالجيزة وتحقيق الإدارة البيئية. لذا نقبل الفرض الرئيسي: "توجد علاقة إرتباطية ذات دلالة معنوية بين تفعيل النظم

الإدارية في حديقة الحيوان بالجيزة وتحقيق الإدارة البيئية". هذا وسوف يساعد الحديقة في الحصول على الأيزو منلما حدث في حديقة حيوان

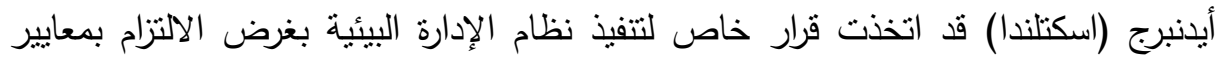
الأيزو العالمية | ..ــا باعتبارها أحد المراكز الرئيسية في أوروبا المعنية بالحفاظ على البيئة وحصلت على الأيزو أبريل 9 . . T (The Zoo, 2010). 


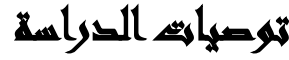

• زيادة عمليات التدريب والتأهيل للإداريين والبيطريين العاملين بالحديقة.

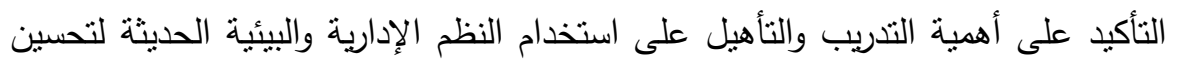
مستوى الأداء، وكذللك أهمية إلمام المدرب المتخصص بالنظور في النظم بما يؤهله لعمليات التدريب والتأهيل للمتقدمين. • العمل على توفير البيانات والمعلومات الصحيحة والاقيقة وتبادلها بين الأفراد والمستويات الإدارية والبيطرية باستخدام الاتصالات الفعالة وذلك لتسهيل عملية الإشراف الرقابي. ه هناك عدة معايير أخلاقية وسلوكية يجب أن تتوفر في البيئة لحماية الحيوان هي:-

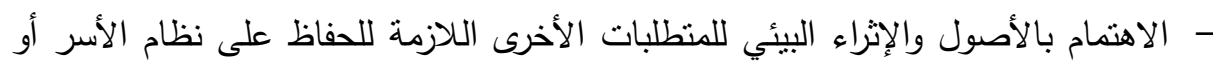

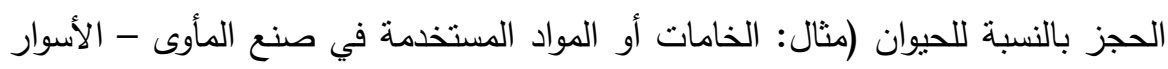

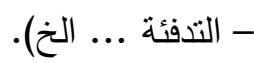

- مراعاة تمييز بعض الأنواع والأجناس ومعاملتها معاملة خاصة كبعض الأجناس المعرضة

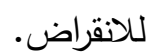
- مراعاة العلاقة بين الحيوانات والبشر القائمين على تلك الحيوانات دون استخلال أو إهمال أو عنف مع الحيوانات.

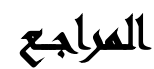

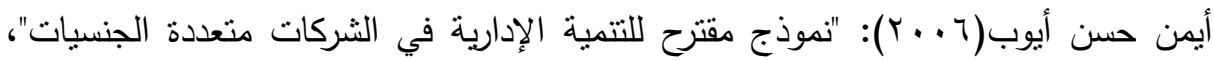
رسالة دكتوراه، كلية التجارة، جامعة عين شمس.

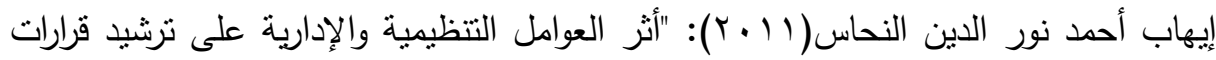
الإدارة البيئية لمواجهة الأزمات"، رسالة ماجستير، معهة الدراسات والبحوث الإدية

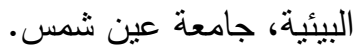

السيد عيد نايل: "الإدارة البيئية ودورها في اتخاذ قرارات التوافق البيئي، مجلة العلوم البيئية"،

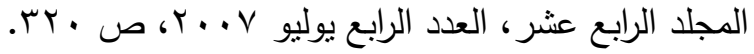




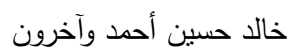

شوقي محمد سليمان(1) (1): "تقييم فاعلية تطبيق نظم الإدارة البيئية في ظل العلاقات

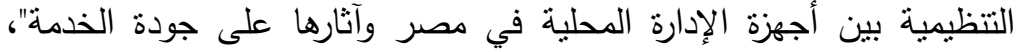

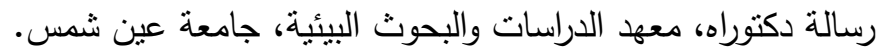

علي السلمي(9/9 (1)): الإدارة العامة ، مكتبة غريب

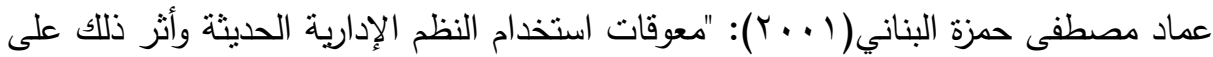

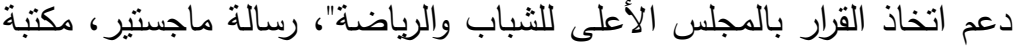

كلية التربية الرياضية بالهرم، جامعة حلوان. لألان.

قلتش عبد الله: اتجاهات حديثة في الفكر الإداري، مجلة إدارة واقتصاد، السنة الخامسة، العدد

$. r+V-r$

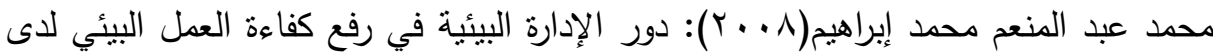

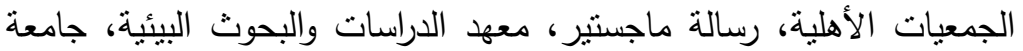

عين شمس.

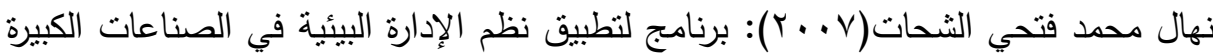

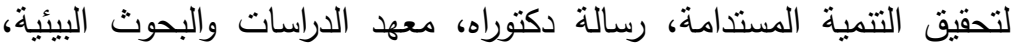
جامعة عين شمس.

An Audit For The Zoo, 8 January 2010, SB Leaders in Environmental Consultancy.

H.; Clauss, M. "Juvenile mortality in captive lesser kudu (Tragelaphus imberbis) at Basle Zoo and its relation to nutrition and husbandry, Journal of Zoo and Wildlife Medicine; 2008. 39(1):86-91. 18ref.

http://elbadil.com/2015/07/13.

M. KILEY-WORTHINGTOPN (2010), "Ecological, Ethological, and Ethically Sound Environments for Animals: Toward Symbiosis, Journal of Agricultural Ethics, Volume 2, pp. 323-347 Printed in the UK. All rights reserved.

North California zoo- United States of America: Environmental Management System.

Viaro, O.; Roberti, F.V.; Bressan, P.M. The efficacy of the education project ZOOSCHOOL- the zoo of Sao Paulo as instrument

المجلد الخامس والثلاثثن، الجزء الثاني، سبتمبر 17 . 


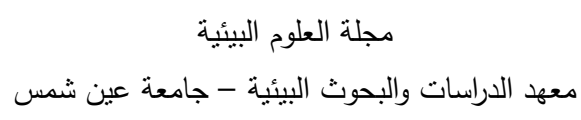

for environments values, [Portuguese]. Veterinaria e Zootecnia; 2011, 18(3):379-383. 12 ref.

http://www.nczoo.org/conservation/atthezoo/EnvironmentalManagem.h tml

\title{
ACTIVATING THE MANAGEMENT SYSTEMS IN THE GIZA ZOO FOR ACHIEVING ENVIRONMENTAL MANAGEMENT
}

\author{
Ahmed, K. H. ${ }^{(1)}$; Salim, M. S. ${ }^{(1)}$ and Metwali, Nahid, I. \\ 1) Faculty of Commerce, Ain Shams University
}

\begin{abstract}
There are several aspects of inconvenience and inappropriateness in dealings and attitudes of human beings with animals. There are also lots of questionings concerning the good appropriate conditions for animals' seizure or being locked in cages or in enclosed environments; and what are the guiding lines for designing management systems for animals; taking into consideration the ecological and local impacts of the management systems of animals within limits of not violating the zoological field.

This study purpose is to activate the environmental management systems in the zoo of Giza, defining also aspects of shortness in environmental planning for improving the environmental performance and assist in decision-making.

The study hypotheses demonstrate that: There is no significant correlation between activation of the administrative systems in the zoo of Giza for achieving environmental management. There is no correlation between applying the environmental management system and improvement of performance level, and improving the control supervision level. The study is concerned with evaluating the current 664

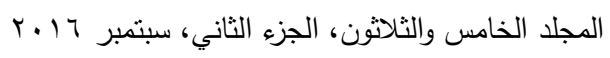


خالد حسين أحمد وآخرون

environmental performance of the environmental management system, defining as well the shortness aspects exist in applying the environmental management system.

The study use the inductive method for preparing the theoretical framework of research, inducing as possible as can the review of literatures, Arabic and foreign references specialized in this domain of environmental management systems in zoos. The study uses the descriptive (qualitative)-analytical method through using an investigation form as the main tool of research, to be administered on workers in the Giza Zoo (224). The study limits on spatial limits embodied in the Giza Zoo and its employees (workers-administrators veterinaries).

Results indicate the following: There is a correlation between applying the environmental management systems and improving performance level in the zoo. There is a correlation between applying the environmental management systems, the supreme administration, planning, and decision-making. There is a significant correlation between applying the environmental management systems and improving the control supervision level.

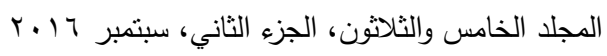

